\title{
Modified Finite Difference and Linear Iterative PDE Methods in Digital Image Inpainting
}

\author{
Sumudu Kalubowila \\ Department of Mathematics, Prairie View A \& M University
}

\begin{abstract}
Image inpainting process is used to develop the damaged image or missing part of the image. This technique has more applications, such as text removal in the image, photo restoration and etc. There are different methods used in image inpainting, such as nonlinear partial differential equations, wavelet transformation, framelet transformation, etc. In this study a linear diffusion PDE method for image inpainting is considered. And to solve this linear PDE a numerical method was developed. Also, different diffusion conductivity, such as constant and nonconstant, were considered for this method. Linear diffusion PDE method was compared with existing non-linear diffusion PDE methods. For an any inpainting method, there exists an error associated with it. So, two different methods were considered to find a relationship between error and inpainting domain.
\end{abstract}

\section{Introduction}

Inpainting has been carried out by professional artists for many years. When done manually, it is a very time-consuming process. The basic idea of this process is to reconstruct damaged parts or missing parts of an image. It has important value in restoration of old photographs; the removal of artifacts in a film; the removal of red eye; the removal of superimposed text; and the removal redundant objects etc. In 2000 SIGGRAPH conference, the idea of digital inpainting was established by Bertalmio-Sapiro-CasellesBallester [1]. Image inpainting has been expanding very fast. It is a very important topic in the field of Digital Image Processing.

Nowadays data exchange has become popular. Since time and skill are required to do image inpainting manually, it is important to find an automatic and fast method. Therefore, different type of successful inpainting techniques were developed in last few years. The idea of the computer algorithm of image inpainting is to fill these missing data with known data surrounding $\mathrm{D}$. $\mathrm{D}$ is the inpainting domain.

\section{Background}

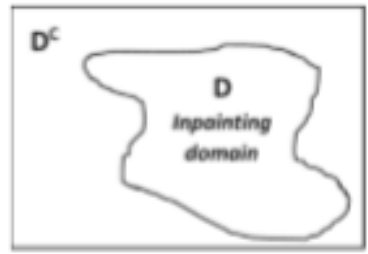

Figure $1 . \mathrm{D}^{\mathrm{c}}$ is the known data and $\mathrm{D}$ is the inpainting domain

\section{Background}

\subsection{Finite difference method}

Laplace equation with Dirichlet boundary condition was considered.

$$
\begin{aligned}
\nabla^{2} u(z) & =\frac{\partial^{2} u}{\partial x^{2}}+\frac{\partial^{2} u}{\partial y^{2}}=0 \quad u \in D \\
u & =f \text { on } \quad \partial D
\end{aligned}
$$

Now consider the square domain with boundary conditions;

$$
A u=F
$$

Where $\mathrm{A}$ is a invertible square matrix. Therefore, value of ui is given by,

$$
u=A^{-1} F
$$

Also, A is a block matrix. So, we can rewrite this matrix as,

$$
A=\left[\begin{array}{lll}
B & I & O \\
I & B & I \\
O & I & B
\end{array}\right]
$$

where, 


$$
\begin{gathered}
B=\left[\begin{array}{ccc}
-4 & 1 & 0 \\
1 & -4 & 1 \\
0 & 1 & -4
\end{array}\right] \quad I=\left[\begin{array}{lll}
1 & 0 & 0 \\
0 & 1 & 0 \\
0 & 0 & 1
\end{array}\right] \\
O=\left[\begin{array}{lll}
0 & 0 & 0 \\
0 & 0 & 0 \\
0 & 0 & 0
\end{array}\right]
\end{gathered}
$$

\subsection{Nonlinear diffusion PDE}

Here we consider the PDE with Neumann boundary condition.

$$
\begin{aligned}
\frac{\partial u}{\partial t} & =-f(u), \quad \text { in } \quad D \quad t \geq 0, \\
u(z, 0) & =u_{0}(z), \quad z \quad \text { in } D, \\
\left.\frac{\partial u}{\partial \mathbf{n}}\right|_{\partial D} & =0,
\end{aligned}
$$

Here $\mathrm{n}$ is the unit inner normal vector. Therefore, numerical solution of this PDE is,

$$
u^{(k+1)}=u^{(k)}-\tau_{k} f\left(u^{(k)}\right) \quad k=0,1,2,3, \ldots
$$

where $\tau_{k}$ is the step size.

Now we consider the heat diffusion equation with heat conductivity $c(|\nabla u|)$.

$$
\begin{aligned}
\frac{\partial}{\partial t} u & =\nabla \cdot(c(|\nabla u|) \nabla u) \quad \text { in } D, t \geq 0 \\
\left.\frac{\partial}{\partial \mathbf{n}} u\right|_{\partial d} & =0 \\
u(z, 0) & =u_{0}(z), \quad z \in D
\end{aligned}
$$

Now apply the anisotropic diffusion equation [3],

$$
\begin{aligned}
& \nabla \cdot(c(|\nabla u|) \nabla u) \\
& \quad=\nabla(c(|\nabla u|) \cdot \nabla u+c(|\nabla u|) \Delta u
\end{aligned}
$$

$u_{0}(z)$ is given by the solution of this PDE,

$$
\begin{aligned}
& \nabla^{2} u(z)=0 \quad u \in D \\
& u=f \quad \text { on } \quad \partial D
\end{aligned}
$$

Now we are going to solve this non-linear diffusion PDE using equation( 2.2.1).

Therefore,

$$
\left.u^{(k+1)}=u^{(k)}-\tau_{k} \nabla \cdot\left(c\left(\mid \nabla u^{(k)}\right) \mid\right) \nabla u^{(k)}\right)
$$

Where, $\mathrm{k}=0,1,2,3, \ldots$

$$
\begin{array}{r}
u^{(1)}=u^{(0)}-\tau_{0} \nabla \cdot\left(c\left(\left|\nabla u^{(0)}\right|\right) \nabla u^{(0)}\right) \\
\text { where } u^{(0)}=u_{0} \\
u^{(2)}=u^{(1)}-\tau_{1} \nabla \cdot\left(c\left(\left|\nabla u^{(1)}\right|\right) \nabla u^{(1)}\right) \\
u^{(3)}=u^{(2)}-\tau_{2} \nabla \cdot\left(c\left(\left|\nabla u^{(2)}\right|\right) \nabla u^{(2)}\right)
\end{array}
$$

We can continue this process and we can get a value of $u$.

\section{Modified finite difference method}

\subsection{Initial value}

When we find the initial value of the inpainting domain, we use five point stencil method. So, in our inpainting domain has $\mathrm{m}$ rows and $\mathrm{n}$ columns. Then our image is look like as in Figure 2.
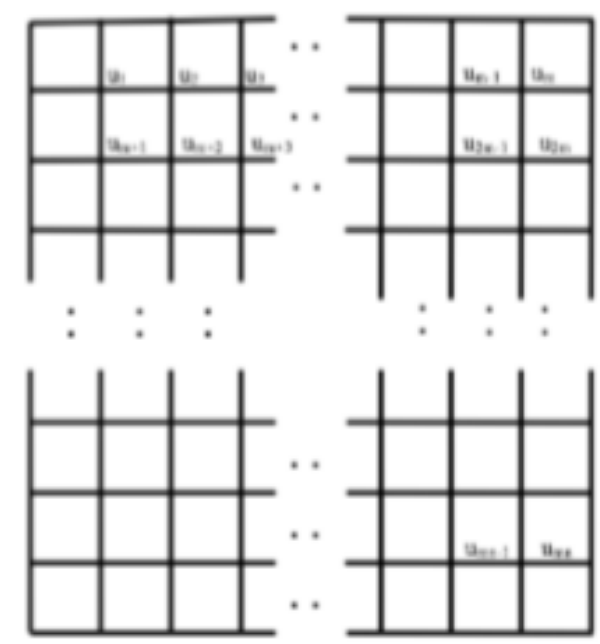

Figure 2. General 2D grid with n rows and m column

Now consider the general matrix for A for size of inpainting domain. 


$$
A=\left[\begin{array}{ccccc}
B & I & O & \ldots & O \\
I & B & I & \ldots & O \\
O & I & B & \ldots & O \\
O & \ldots & \ldots & \ldots & O \\
& \ldots & \ldots & \ldots & \ldots \\
& \ldots & \ldots & \ldots & \ldots \\
& \ldots & I & B & I \\
& \ldots & \ldots & \ldots B & I
\end{array}\right]
$$

We have the boundary in formation and we have to find $u 1, \ldots, u 9$. Normal 5-point method we need only one adjacent boundary level data. But in this method, we need two adjacent boundary level data to fill inside data.

\section{Step 1:}

\begin{tabular}{|c|c|c|c|c|c|c|}
\hline B1 & B2 & B3 & B4 & B5 & B6 & B7 \\
\hline B8 & B9 & B10 & B11 & B12 & B13 & B14 \\
\hline B15 & B16 & U1 & U2 & U3 & B17 & B18 \\
\hline B19 & B20 & U4 & & U6 & B21 & B22 \\
\hline B23 & B24 & U7 & U8 & U9 & B25 & B26 \\
\hline B27 & B28 & B29 & B30 & B31 & B32 & B33 \\
\hline B34 & B35 & B36 & B37 & B38 & B39 & B40 \\
\hline
\end{tabular}

Figure 4. 5-point apply to level 1

$B$ is a $\mathrm{m} \times \mathrm{m}$ matrix. Where $m$ is a number of columns in inside of the grid.

$$
\begin{aligned}
& I=\left(\begin{array}{lllll}
1 & 0 & & & \\
0 & 1 & 0 & & \\
& & \ddots & & \\
& & 0 & 1 & 0 \\
& & & 0 & 1
\end{array}\right) \\
& O=\left(\begin{array}{lllll}
0 & 0 & & & \\
0 & 0 & 0 & & \\
& & \ddots & & \\
& & 0 & 0 & 0 \\
& & & 0 & 0
\end{array}\right)
\end{aligned}
$$

When we apply this method to our image, we did some development. We did image inpainting technique level by level. That is;

\begin{tabular}{|c|c|c|c|c|c|c|}
\hline B1 & B2 & B3 & B4 & B5 & B6 & B7 \\
\hline B8 & B9 & B10 & B11 & B12 & B13 & B14 \\
\hline B15 & B16 & U1 & U2 & U3 & B17 & B18 \\
\hline B19 & B20 & C4 & U5 & U6 & B21 & B22 \\
\hline B23 & B24 & U7 & U8 & C9 & B25 & B26 \\
\hline B27 & B28 & B29 & B30 & B31 & B32 & B33 \\
\hline B34 & B35 & B36 & B37 & B38 & B39 & B40 \\
\hline
\end{tabular}

Figure 3. 5-point apply level by level
Using the boundary data (red data) we can calculate adjacent level data (green data). Here we apply 5-point stencil method in different way;

$$
\begin{aligned}
& \text { For } U_{2} \\
& \qquad U_{2}=4 B_{11}-B_{4}-B_{10}-B_{12}
\end{aligned}
$$

For $U_{4}$

$$
U_{4}=4 B_{20}-B_{16}-B_{19}-B_{24}
$$

For $U_{6}$

$$
U_{6}=4 B_{21}-B_{17}-B_{22}-B_{25}
$$

For $U_{8}$

$$
U_{8}=4 B_{30}-B_{29}-B_{31}-B_{37}
$$

\section{Step 2:}

We use two different formulas for corner points and consider the average value. Such as;

For $U_{1}$

$$
\begin{aligned}
U_{1}^{1} & =4 B_{16}-B_{9}-B_{15}-B_{20} \\
U_{1}^{2} & =4 B_{10}-B_{3}-B_{9}-B_{11} \\
U_{1} & =\frac{U_{1}^{1}+U_{1}^{2}}{2}
\end{aligned}
$$

For $U_{3}$

$$
\begin{aligned}
U_{3}^{1} & =4 B_{12}-B_{5}-B_{11}-B_{13} \\
U_{3}^{2} & =4 B_{17}-B_{13}-B_{18}-B_{21} \\
U_{3} & =\frac{U_{3}^{1}+U_{3}^{2}}{2}
\end{aligned}
$$


For $U_{7}$

$$
\begin{aligned}
U_{7}^{1} & =4 B_{24}-B_{20}-B_{23}-B_{28} \\
U_{7}^{2} & =4 B_{29}-B_{28}-B_{30}-B_{36} \\
U_{7} & =\frac{U_{7}^{1}+U_{7}^{2}}{2}
\end{aligned}
$$

For $U_{9}$

$$
\begin{aligned}
& U_{9}^{1}=4 B_{25}-B_{21}-B_{26}-B_{32} \\
& U_{9}^{2}=4 B_{31}-B_{30}-B_{32}-B_{38} \\
& U_{9}=\frac{U_{9}^{1}+U_{9}^{2}}{2}
\end{aligned}
$$

\section{Step 3:}

When we are finding next level of data (blue data), we use the adjacent level data (green data);

$$
U_{5}=\frac{U_{2}+U_{4}+U_{6}+U_{8}}{4}
$$

\begin{tabular}{|c|c|c|c|c|c|c|}
\hline B1 & B2 & B3 & B4 & B5 & B6 & B7 \\
\hline B8 & B9 & B10 & B11 & B12 & B13 & B14 \\
\hline B15 & B16 & U1 & U2 & U3 & B17 & B18 \\
\hline B19 & B20 & U4 & U5 & U6 & B21 & B22 \\
\hline B23 & B24 & U7 & U8 & U9 & B25 & B26 \\
\hline B27 & B28 & B29 & B30 & B31 & B32 & B33 \\
\hline B34 & B35 & B36 & B37 & B38 & B39 & B40 \\
\hline
\end{tabular}

Figure 5. 5-point apply to level 2

Now we compare our method with the existing method.

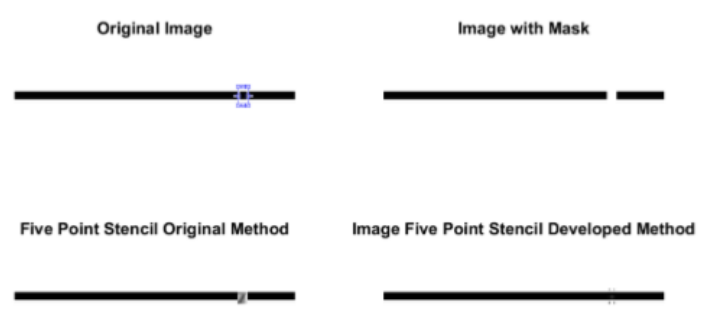

Figure 6. Standard 5-point method and modified 5-point method

When we check the two different approaches of 5 point stencil methods, we can see modified method
PSNR value is larger than that of the standard 5-point stencil method. So, we use the modified 5-point stencil method values for our further calculations.

Table 1. PSNR value for standard 5-point method and modified 5-point method

\begin{tabular}{ccc}
\hline & $\begin{array}{c}\text { Standard } \\
\text { 5-point method }\end{array}$ & $\begin{array}{c}\text { Modified } \\
\text { 5-point method }\end{array}$ \\
\hline PSNR & 29.1787 & 39.2958 \\
\hline
\end{tabular}

see modified method PSNR value is larger than that of the standard 5-point stencil method. So, we use the modified 5-point stencil method values for our further calculations.

\section{Linear iterative PDE method}

In 2009, [2] C. K. Chui developed a multiresolution approximation method for image inpainting and surface completion. Here they use partial differential equation of anisotropic diffusion to know data.

$$
\begin{aligned}
\frac{\partial}{\partial t} u_{j} & =\nabla \cdot\left(c\left(\left|\nabla u_{j-1}\right|\right) \nabla u_{j}\right) \text { in } D, t \geq 0 \\
\left.\frac{\partial}{\partial \mathbf{n}} u_{j}\right|_{\partial d} & =0 \\
u_{j}(z, 0) & =u_{0}(z), \quad z \in D
\end{aligned}
$$

Where $\mathrm{j}=1,2, \ldots .$. and $c\left(\left|\nabla u^{j}(z)\right|\right.$ is the diffusion conductivity.

Here we have a set of linear partial differential equations. This is also called local image inpainting method.

Now we are going to solve this linear iterative equations.

when $\mathrm{j}=1$;

$$
\begin{aligned}
\frac{\partial}{\partial t} u_{1} & =\nabla \cdot\left(c\left(\left|\nabla u_{0}\right|\right) \nabla u_{1}\right) \text { in } D, t \geq 0 \\
\left.\frac{\partial}{\partial \mathbf{n}} u_{1}\right|_{\partial d} & =0 \\
u_{1}(z, 0) & =u_{0}(z), \quad z \in D
\end{aligned}
$$

Now apply the equation for this PDE,

$$
u_{1}^{(k+1)}=u_{1}^{(k)}-\tau_{k} f\left(u_{1}^{(k)}\right) \quad k=0,1,2,3, \ldots
$$

Now $f\left(u_{1}\right)=\nabla \cdot\left(c\left(\left|\nabla u_{0}\right|\right) \nabla u_{1}\right)$

Therefore,

$$
\begin{array}{r}
u_{1}^{(k+1)}=u_{1}^{(k)}-\tau_{k} \nabla \cdot\left(c\left(\left|\nabla u_{0}\right|\right) \nabla u_{1}^{(k)}\right) \\
k=0,1,2,3, \ldots
\end{array}
$$




$$
\begin{aligned}
& \text { when } \quad \mathrm{k}=0 ; \\
& \begin{array}{r}
u_{1}^{(1)}=u_{1}^{(0)}-\tau_{0} \nabla \\
\cdot\left(c\left(\left|\nabla u_{0}\right|\right) \nabla u_{1}^{(0)}\right) \\
\text { where } u_{1}^{(0)}=u_{0}
\end{array}
\end{aligned}
$$

when $\mathrm{k}=1$;

$$
u_{1}^{(2)}=u_{1}^{(1)}-\tau_{1} \nabla \cdot\left(c\left(\left|\nabla u_{0}\right|\right) \nabla u_{1}^{(1)}\right)
$$

when $\mathrm{k}=2$;

$$
u_{1}^{(3)}=u_{1}^{(2)}-\tau_{2} \nabla \cdot\left(c\left(\left|\nabla u_{0}\right|\right) \nabla u_{1}^{(2)}\right)
$$

We can continue this process and we can get a value of $u_{1}$. We use this value to find $u_{2}$.

when $\mathrm{j}=2$;

$$
\begin{aligned}
\frac{\partial}{\partial t} u_{2} & =\nabla \cdot\left(c\left(\left|\nabla u_{1}\right|\right) \nabla u_{2}\right) \text { in } D, t \geq 0 \\
\left.\frac{\partial}{\partial \mathbf{n}} u_{2}\right|_{\partial d} & =0 \\
u_{2}(z, 0) & =u_{0}(z), \quad z \in D
\end{aligned}
$$

Now consider $f\left(u_{2}\right)=\nabla \cdot\left(c\left(\left|\nabla u_{1}\right|\right) \nabla u_{2}\right)$ Therefore,

$$
\begin{array}{r}
u_{2}^{(k+1)}=u_{2}^{(k)}-\tau_{k} \nabla \cdot\left(c\left(\left|\nabla u_{1}\right|\right) \nabla u_{2}^{(k)}\right) \\
k=0,1,2,3, \ldots
\end{array}
$$

When $\mathrm{k}=0$;

$$
\begin{array}{r}
u_{2}^{(1)}=u_{2}^{(0)}-\tau_{0} \nabla \cdot\left(c\left(\left|\nabla u_{1}\right|\right) \nabla u_{2}^{(0)}\right) \\
\text { where } u_{2}^{(0)}=u_{1}
\end{array}
$$

when $\mathrm{k}=1$;

$$
u_{2}^{(2)}=u_{2}^{(1)}-\tau_{1} \nabla \cdot\left(c\left(\left|\nabla u_{1}\right|\right) \nabla u_{2}^{(1)}\right)
$$

when $\mathrm{k}=2$;

$$
u_{2}^{(3)}=u_{2}^{(2)}-\tau_{2} \nabla \cdot\left(c\left(\left|\nabla u_{1}\right|\right) \nabla u_{2}^{(2)}\right)
$$

We can continue this process and we can get a value of $u_{2}$. We use this value to find $u_{3}$.

$$
\text { when } \mathrm{j}=3 \text {; }
$$

$$
\begin{aligned}
\frac{\partial}{\partial t} u_{3} & =\nabla \cdot\left(c\left(\left|\nabla u_{2}\right|\right) \nabla u_{3}\right) \text { in } D, t \geq 0 \\
\left.\frac{\partial}{\partial \mathbf{n}} u_{3}\right|_{\partial d} & =0 \\
u_{3}(z, 0) & =u_{0}(z), \quad z \in D
\end{aligned}
$$

Now consider $f\left(u_{3}\right)=\nabla \cdot\left(c\left(\left|\nabla u_{2}\right|\right) \nabla u_{3}\right)$ Therefor,

$$
\begin{array}{r}
u_{3}^{(k+1)}=u_{3}^{(k)}-\tau_{k} \nabla \cdot\left(c\left(\left|\nabla u_{2}\right|\right) \nabla u_{3}^{(k)}\right) \\
k=0,1,2,3, \ldots
\end{array}
$$

when $\quad \mathrm{k}=0$;

$$
\begin{array}{r}
u_{3}^{(1)}=u_{3}^{(0)}-\tau_{0} \nabla \cdot\left(c\left(\left|\nabla u_{2}\right|\right) \nabla u_{3}^{(0)}\right) \\
\text { where } u_{3}^{(0)}=u_{2}
\end{array}
$$

when $\mathrm{k}=1$;

$$
u_{3}^{(2)}=u_{3}^{(1)}-\tau_{1} \nabla \cdot\left(c\left(\left|\nabla u_{2}\right|\right) \nabla u_{3}^{(1)}\right)
$$

when $\mathrm{k}=2$;

$$
u_{3}^{(3)}=u_{3}^{(2)}-\tau_{2} \nabla \cdot\left(c\left(\left|\nabla u_{2}\right|\right) \nabla u_{3}^{(2)}\right)
$$

We can continue this process and we can get a value of u3. Using this method, we can solve linear iterative PDE.

Initial value of the non-linear and linear iterative PDE method is the 5-point stencil method values. Using a MATLAB program, we inpainted the damaged image. Here we compared MATLAB out puts for different in painting methods with different diffusion conductivity.

When we compare the inapinted image with the original image, we use PSNR values. Which is Peak Signal Noise Ratio. We define PSNR using mean squared error (MSE) and formula is given by,

$$
P S N R=20 \cdot \log _{10} \frac{M A X}{\sqrt{M S E}} .
$$

Where MAX is the maximum possible pixel value of the image.

Case 1: Linear iterative and nonlinear image inpainting PDE with constant diffusion conductivity

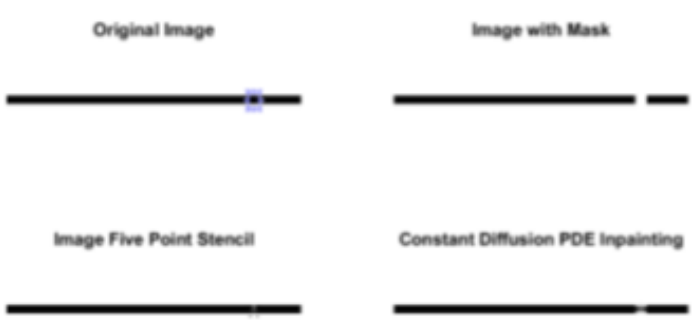

Figure 7. Image inpainting methods with constant diffusion 
Table 2: PSNR value for linear iterative PDE and non-linear image inpainting PDE with constant diffusion conductivity

\begin{tabular}{ccc}
\hline $\begin{array}{c}\text { Inpainting } \\
\text { methods }\end{array}$ & $\begin{array}{c}\text { 5-point stencil } \\
\text { method }\end{array}$ & $\begin{array}{c}\text { Constant } \\
\text { conductivity } \\
\text { PDE }\end{array}$ \\
\hline PSNR & 35.5747 & 29.9493 \\
\hline
\end{tabular}

Here we consider the constant conductivity. That is,

$c(p)=c$. When $c$ is a constant there is a no difference between linear iterative PDE and nonlinear PDE.

That is, Linear iterative PDE,

$$
\left.\frac{\partial}{\partial t} u_{i}=\nabla \cdot(c) \nabla u_{i}\right)=c \Delta u_{i}
$$

\section{Non-linear PDE,}

$$
\left.\frac{\partial}{\partial t} u=\nabla \cdot(c) \nabla u\right)=c \Delta u
$$

When $\mathrm{c}$ is a constant, we have worst inpainted image. Therefore 5-point stencil method is better than the constant diffusion conductivity PDE.

Case 2: Linear iterative PDE and non-linear image inpainting PDE with inverse proportional diffusion conductivity



Non-Linear PDE Inpainting Linear Iterative PDE Image

Figure 8. Image inpainting methods with inverse proportional diffusion conductivity

Here we consider the inverse proportional conductivity. That is,

$\mathrm{c}(\mathrm{p})=\frac{1}{p}$.

With this diffusion conductivity,diffusion PDE is called TV inpainting method.

When we use this in MATLAB , we consider

$c(p)=\frac{1}{\varepsilon+p}$. Because we want to ignore the value of $\mathrm{p}=0$.
Table 3: PSNR value for linear iterative PDE and non-linear image inpainting PDE with inverse proportional diffusion conductivity

\begin{tabular}{cccc}
\hline $\begin{array}{c}\text { Inpainting } \\
\text { methods }\end{array}$ & $\begin{array}{c}\text { 5-point } \\
\text { stencil } \\
\text { method }\end{array}$ & $\begin{array}{c}\text { Non-linear } \\
\text { PDE }\end{array}$ & $\begin{array}{c}\text { Linear } \\
\text { iterative } \\
\text { PDE }\end{array}$ \\
\hline PSNR & 30.3192 & 26.3699 & 31.9985 \\
\hline
\end{tabular}

We use the 5-point stencil values as an initial value of Linear iterative PDE and nonlinear PDE. When we study this table, we can see PSNR value is increase to 5-point stencil method to linear iterative PDE method. Also, PSNR value of 5-point stencil method to linear iterative PDE method is decreasing. That is our inpainted image is worse than the 5point stencil method inpainted image. Therefore, linear iterative PDE method gave a better inpainted image.

Case 3: Linear iterative PDE and non-linear image inpainting PDE with Gaussian diffusion conductivity

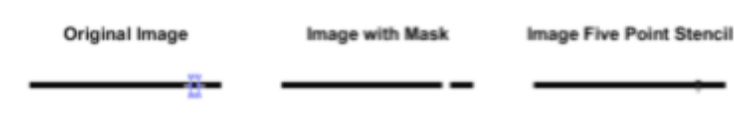

Non-Linear PDE Inpainting Linear Iterative PDE Image

Figure 9. Image inpainting methods with Gaussian diffusion conductivity

Table 4. PSNR value for linear iterative PDE and non-linear image inpainting PDE with Gaussian proportional diffusion conductivity

\begin{tabular}{cccc}
\hline $\begin{array}{c}\text { Inpainting } \\
\text { methods }\end{array}$ & $\begin{array}{c}\text { 5-point } \\
\text { stencil } \\
\text { method }\end{array}$ & $\begin{array}{c}\text { Non-linear } \\
\text { PDE }\end{array}$ & $\begin{array}{c}\text { Linear } \\
\text { iterative } \\
\text { PDE }\end{array}$ \\
\hline PSNR & 29.9851 & 27.0343 & 33.1426 \\
\hline
\end{tabular}

Here we consider the Gaussian Diffusion Conductivity. That is,

$$
\mathrm{c}(\mathrm{p})=e^{-\frac{p^{2}}{k^{2}}} \text {. }
$$

Here also, we use the5-pointstencilvalues as an initial value of linear iterative PDE and non-linear PDE.

When we study this table, we can see PSNR value is increased to 5-point stencil method to linear iterative PDE method. But PSNR value of 5-point 
stencil method is decreased in the non-linear PDE method. That is non-linear method given worst inpainted image. Therefore, linear iterative PDE method gave the best inpainted image

Case 4: Linear iterative PDE and non-linear image inpainting PDE with Lorentz diffusion conductivity



Non-Linear PDE Inpainting Linear Iterative PDE Image

Figure 10. Image inpainting methods with Lorentz diffusion conductivity

Table 5. PSNR value for linear iterative PDE and non-linear image inpainting PDE with Lorentz proportional diffusion conductivity

\begin{tabular}{cccc}
\hline $\begin{array}{c}\text { Inpainting } \\
\text { methods }\end{array}$ & $\begin{array}{c}5 \text {-point } \\
\text { stencil } \\
\text { method }\end{array}$ & $\begin{array}{c}\text { Non-linear } \\
\text { PDE }\end{array}$ & $\begin{array}{c}\text { Linear } \\
\text { iterative } \\
\text { PDE }\end{array}$ \\
\hline PSNR & 34.7837 & 29.9902 & 37.3648 \\
\hline
\end{tabular}

Here we consider the Lorentz Diffusion Conductivity. That is,

$$
\mathrm{c}(\mathrm{p})=\frac{1}{1+\frac{p^{2}}{k^{2}}} \text {. }
$$

Here also, we use the 5-pointstencilvalues as a initial value of linear iterative PDE and non-linear PDE. When we study this table, we have same idea of previous methods. That is linear iterative PDE method gave the best inpainted image.

\section{Conclusion}

In this paper we solved Poisson's equation using 5-point stencil method. Here we used modified 5point stencil method. It gave better results than the standard 5-point stencil method. Using modified 5point stencil method values as an initial condition, we solve liner iterative and non-linear diffusion PDE. Also, here we consider different diffusion conductivity and compare their results. Using a PSNR value, we can see Linear iterative diffusion PDE method given the better results. Also, constant diffusion conductivity PDE had the worst results. Inverse diffusion conductivity PDE is better than that of the constant diffusion PDE. But it is worse than that of the Gaussian and Lorentz diffusion conductivity
PDE. Gaussian and Lorentz diffusion conductivity Linear iterative PDE gave the better results for image inpainting.

\section{References}

[1] Bertalmio, M., Sapiro, G., Caselles, V., Ballester, C., (2000). Image inpainting. SIGGRAPH '00 Proceedings of the 27th annual conference on Computer graphics and interactive techniques, Wesley Publishing Co., NewYork, NY, USA, pages 417-424, 2000.

[2] Chui, C.K., (2009). An MRA approach to surface completion and image inpainting, Appllied and Computational Harmonic Analysis, Elsevier Science, Vol. 26 , pages $270-276$.

[3] Perona, P., Malik, J., (1990). Scale space and edge detection using anistropic diffusion, IEEE PAML, Vol. 12, pages $629-639$. 